\title{
HYDRO-ELASTIC OSCILLATIONS OF THE BOTTOM MEMBRANE OF A RECTANGULAR CONTAINER FILLED WITH FLUID
}

\author{
MANUEL GASCÓN-PÉREZ \\ Escuela Técnica Superior de Ingeniería Aeronáutica y del Espacio \\ Universidad Politécnica de Madrid \\ 28040 Madrid, Spain \\ manuel.gascon@upm.es
}

\begin{abstract}
The analysis of the hydro-elastic interactions of the covering membrane of fluid-filled cavities or containers has a main importance due to the solution of practical problems founded in engineering applications. In this paper the dynamic behaviour of the bottom membrane of a rectangular container filled with a non-viscous and incompressible fluid is analyzed. The fluid velocity potential is obtained first by applying a method of separation of variables and afterwards the pressure field is calculated with the momentum's linearized equation. Taking into account the deformation equation for the membrane in contact with the fluid and by applying a discretization procedure to the associated generalized work equation, a system is obtained, for the calculus of the membrane frequencies of vibration. The influence of different geometrical parameters such as dimension, aspect ratio, container relative height, relative thickness as well as the fluid density on these frequencies is analysed. Validation of the method is made by comparing the results with those obtained by other authors and theories.
\end{abstract}

\section{Introduction}

The fluid-structure interaction concerning oscillating membranes is interesting because of the wide variety of technological applications such as space reflector in satellites, biological prosthesis, see Jenkins [1], or devices common in micro electromechanical systems such as micropumps, see Laser et al [2], Pan et al [3] or Shabani et al [4], ultrasonic transducers, see Jin et al [5] and pressure sensors, see Defay et al [6]. The performances of these microsystems are directly dependent upon properly controlling the resonant frequencies because for example, micropumps operates at the resonant frequency with more efficiency and so, thus reducing power requirements. On the other hand, there are applications such as liquid containers of airplanes, missiles or space vehicles in which is interesting to know the frequencies of the coupled system. In many cases the liquid frequencies are close to other frequencies of the system resulting in a disturbance of the motion and even instability and failure so that a shifting of these frequencies is a way of solving the problem. This is done by covering the free liquid surface with a flexible structural member, such as a membrane or a thin elastic plate.

The problem of the fluid-membrane interactions has been investigated by many researchers. Cheung and Zhou [7] studied the natural frequencies of an elastic thin plate into a rectangular hole and connected to the rigid bottom slab of a rectangular container filled with fluid having a free surface and assumed to be incompressible, inviscid and irrotational employing an analytical Ritz method to study the vibratory characteristics of the plate in contact with the fluid. Daneshmand and Ghavanloo [8] investigated the two dimensional couple free vibrations of a fluid filled rectangular container with a sagged bottom membrane which consists of two rigid walls and a membrane 
anchored along two rigid vertical walls, and is filled with incompressible and inviscid fluid. Along the contact surface between the bottom membrane and the fluid, the compatibility requirement was applied for the fluid-structure interactions and the finite element method was used to calculate the natural frequencies and mode shapes of the fluid-membrane system. Bauer [9] \& [10] analyzed the hydro-elastic vibrations in a cylindrical and rectangular containers with the free liquid surface covered by a flexible membrane or a thin elastic plate using a variational formulation . Chiba et al .[11] considered the free hydroelastic vibration in a cylindrical container with a bottom membrane, containing liquid with surface tension. Tariverdilo et al. [12,13] considered the free vibration of a circular plate or membrane located at the bottom of a cylindrical container filled with an incompressible and non-viscous fluid, for which it applies a variational approach taking into account the deformation of the plate and the velocity potential function for the fluid also including its boundary conditions. Finally Gascón-Pérez [18] that analyzes the problem of interaction of an oscillating rectangular membrane submerged in an infinite compressible fluid domain by a boundary element method, calculating the change in the natural frequencies of an elastic rectangular membrane in contact with fluid with respect to the vacuum case, and in [17], the same author analyzes the acoustic influence on the vibration of a cylindrical membrane drum filled up with a compressible fluid, employing a boundary element method.

The vibration of a bottom membrane of a rectangular container filled with incompressible and non-viscous fluid is analyzed in this paper. Taking into account the fluid velocity potential and the momentum's linearized equation for the fluid pressure, considering the equation of motion of the membrane and applying an associated generalized work equation, the frequencies of the coupled system are evaluated by a collocation method, leading to an eigenvalue problem. This method is characterized by its relative simplicity.

Finally, the effects of geometrical parameters such as container relative height, rectangular membrane dimension and aspect ratio, and fluid parameters such as density on the free vibration response of the coupled system are investigated. Validation of the method is made by comparing the results with those obtained by other authors and theories.

\section{Problem Formulation}

The dynamic response of the bottom membrane of a rectangular container is governed by the equation, see Wang [14]:

$$
T \Delta \delta(x, y, t)-\rho_{m} t_{h} \frac{\partial^{2} \delta}{\partial t^{2}}=p(x, y, t)
$$

with $\delta$ the membrane deformation, $T$ the membrane tension, $t_{h}$ the membrane thickness, and $\rho_{m}$ the material density of the membrane.

It is supposed that the only action exerted on the flexible membrane is the fluid pressure $p$ inside the rectangular container and that it is in contact with it.

Considering the contact between the membrane and the fluid, the boundary condition for the momentum equation is obtained by:

$$
\frac{\partial p}{\partial z}=-\rho_{f} \frac{\partial^{2} \delta}{\partial t^{2}} \text {. at } z=H \quad \text { and } 0<x<a, \quad 0<y<b
$$

For a membrane placed at $z=H$, and with $a, b$ the side lengths of the membrane, being $H$ the container height, see Fig.1 
If it is supposed an oscillating motion for both, the fluid and the membrane, the solutions for the deformation and fluid pressure takes the form:

$$
\delta(x, y, t)=\tilde{\delta}(x, y) \cdot e^{i \omega t} . \text { and } p(x, y, z, t)=\tilde{p}(x, y, z) \cdot e^{i \omega t}
$$

The deflection of the membrane, when coupled with the fluid, will be expressed as a linear combination of the normal modes of the membrane in vacuum:

$$
\delta(x, y, t)=\sum_{m} \sum_{n} \delta_{m}^{n}(x, y) \cdot \bar{d}_{m}^{n} \cdot e^{i \omega_{m}^{n} t}
$$

Where $\bar{d}_{m}^{n}$ are the weight coefficients associated to each mode that contribute to the total deformation of the rectangular membrane in contact with fluid.

The functions $\delta_{m}^{n}(x, y)$ for a rectangular membrane clamped along its edges, have the general expression see Wang [14]:

$$
\delta_{m}^{n}(x, y)=\sin \left(\frac{m \pi x}{a}\right) \cdot \sin \left(\frac{n \pi y}{b}\right)
$$

The indexes $m-1, n-1$ represent the number of nodal lines in the $y, x$ directions respectively.

And for the rigid walls $\frac{\partial p}{\partial n}=0$, with $\vec{n}$ the normal to the surface of the wall.

In Fig. 1 the geometrical characteristics of the rectangular container are represented, in order to be defined and clarified.

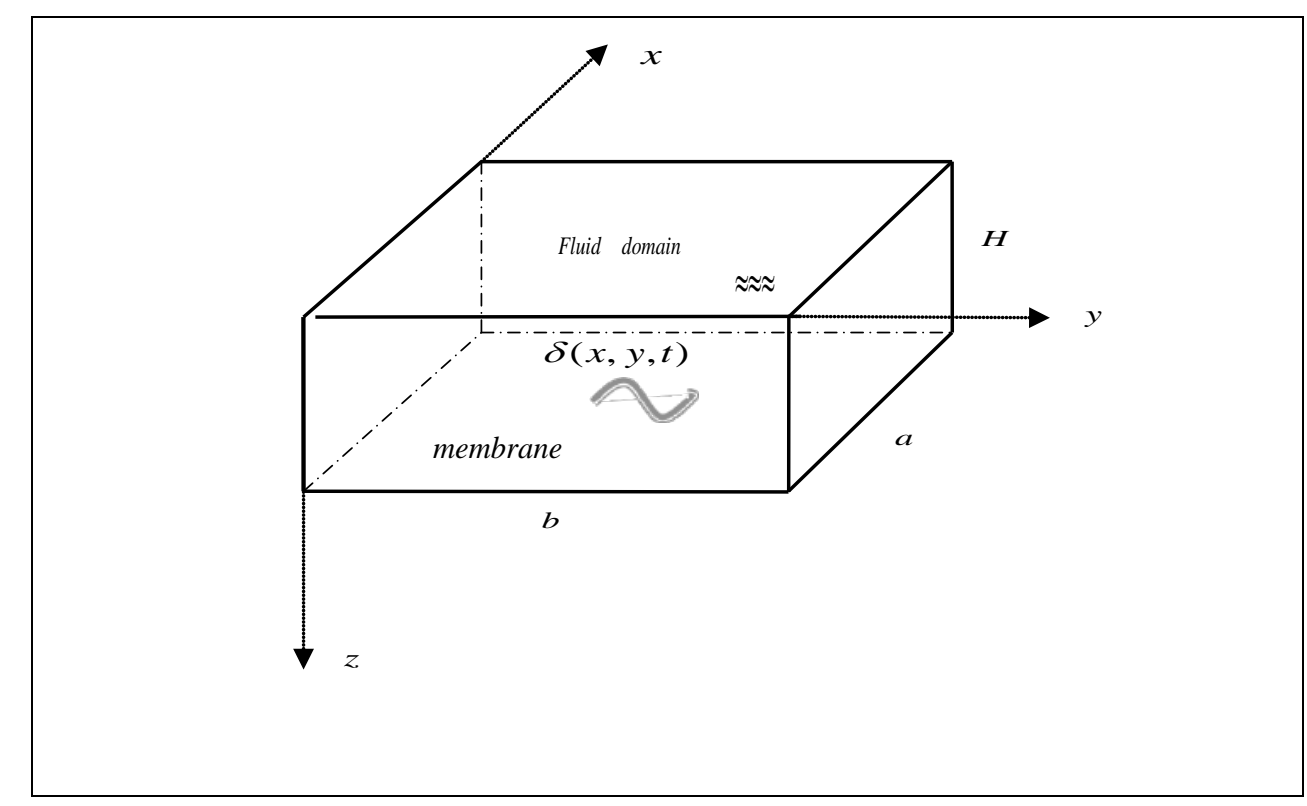

Fig. 1. Scheme of the membrane rectangular container showing the different geometrical parameters

\section{Calculus of the pressure over the membrane}


Assuming incompressible and inviscid fluid, the fluid motion induced by the vibration of the rectangular membrane is described using the velocity potential function, Munson [15]. This velocity potential $\varphi(x, y, z, t)$ satisfies the equation:

$$
\Delta \varphi=0
$$

being $\Delta$ the Laplace operator, together with the boundary conditions:

- For the rigid wall boundaries, $\frac{\partial \varphi}{\partial n}=0$, being $\vec{n}$ the normal to the wall surface, i.e.:

$$
\begin{aligned}
& \frac{\partial \varphi}{\partial x}=0 \quad \text { for } \quad x=0, a \quad 0 \leq y \leq b \quad 0 \leq z \leq H \\
& \frac{\partial \varphi}{\partial y}=0 \quad \text { for } \quad y=0, b \quad 0 \leq x \leq a \quad 0 \leq z \leq H . \\
& \frac{\partial \varphi}{\partial z}=0 \text { for } \quad z=0 \quad 0 \leq x \leq a \quad 0 \leq y \leq b .
\end{aligned}
$$

- For the bottom membrane boundary:

$$
\frac{\partial \varphi}{\partial z}=\frac{\partial \delta}{\partial t} \text { for } \quad z=H \quad 0 \leq x \leq a \quad 0 \leq y \leq b
$$

Applying a method of separation of variables in space-time and expanding in series, the velocity potential function and the membrane deformation are expressed:

$$
\begin{aligned}
& \varphi(x, y, z, t)=\sum_{m} \sum_{n} \varphi_{m}^{n}(x, y, z) \cdot f_{m}^{n}(t) . \\
& \delta(x, y, t)=\sum_{m} \sum_{n} \delta_{m}^{n}(x, y) \cdot d_{m}^{n}(t) .
\end{aligned}
$$

where for harmonic motion $f_{m}^{n}(t)=\bar{f}_{m}^{n} \cdot e^{i \omega_{m}^{n} t}$. and $d_{m}^{n}(t)=\bar{d}_{m}^{n} \cdot e^{i \omega_{m}^{n} t}$.

Considering Eq.6 for the velocity potential and the boundary conditions Eq.7, Eq.8 and Eq.9, and applying again separation of variables in space coordinates, the solution takes this form:

$$
\varphi(x, y, z, t)=\sum_{m} \sum_{n} \sin \left(\frac{m \pi x}{a}\right) \cdot \sin \left(\frac{n \pi y}{b}\right) \cosh \left(\lambda_{m}^{n} z\right) \cdot f_{m}^{n}(t) .
$$

Where the function $\varphi_{m}^{n}(x, y, z)=\delta_{m}^{n}(x, y) \cdot \cosh \left(\lambda_{m}^{n} z\right)$. and the parameter $\lambda_{m}^{n}=\sqrt{\left(\frac{m \pi}{a}\right)^{2}+\left(\frac{n \pi}{b}\right)^{2}}$. 
Taking into account the boundary condition, Eq.10, of the fluid-membrane interface, the expression for the fluid velocity potential, Eq.14 and the membrane deformation, Eq.12, the following expression is obtained:

$$
\sum_{m} \sum_{n} \delta_{m}^{n}(x, y) \sinh \left(\lambda_{m}^{n} H\right) \lambda_{m}^{n} f_{m}^{n}(t)=\sum_{m} \sum_{n} \delta_{m}^{n}(x, y) \dot{d}_{m}^{n}(t)
$$

from where it is deduced:

$$
f_{m}^{n}(t)=\frac{1}{\lambda_{m}^{n} \sinh \left(\lambda_{m}^{n} H\right)} \dot{d}_{m}^{n}(t)
$$

To evaluate the fluid pressure field, without considering the effects of gravity and assuming small displacements, the momentum's linearized equation is taken into account, Munson [15]:

$$
\frac{\partial \varphi}{\partial t}+\frac{p}{\rho_{f}}=0
$$

Considering the Eq.14 for the velocity potential and Eq.17, the fluid pressure field is deduced:

$$
p(x, y, z, t)=-\rho_{f} \sum_{m} \sum_{n} \delta_{m}^{n}(x, y) \cosh \left(\lambda_{m}^{n} z\right) \dot{f}_{m}^{n}(t) .
$$

By substituting (2.16) in (2.18), the fluid pressure over the membrane $z=H$ is:

$$
p(x, y, H, t)=-\rho_{f} \sum_{m} \sum_{n} \frac{\cot h\left(\lambda_{m}^{n} H\right)}{\lambda_{m}^{n}} \delta_{m}^{n}(x, y) \ddot{d}_{m}^{n}(t) .
$$

And considering Eq.13 for harmonic motion and therefore $\ddot{d}_{m}^{n}(t)=-\omega_{m}^{n 2} \bar{d}_{m}^{n} e^{i \omega_{m}^{n} t}$. :

$$
p(x, y, H, t)=\rho_{f} \sum_{m} \sum_{n} \frac{\cot h\left(\lambda_{m}^{n} H\right)}{\lambda_{m}^{n}} \omega_{m}^{n 2} \delta_{m}^{n}(x, y) \bar{d}_{m}^{n} e^{i \omega_{m}^{n} t} .
$$

that can be expressed as

$$
p(x, y, H, t)=\sum_{m} \sum_{n} P_{m}^{n}(x, y) \bar{d}_{m}^{n} e^{i \omega_{m}^{n} t} .
$$

being $P_{m}^{n}$, the pressure mode:

$$
P_{m}^{n}(x, y)=\rho_{f} \frac{\cot h\left(\lambda_{m}^{n} H\right)}{\lambda_{m}^{n}} \omega_{m}^{n 2} \delta_{m}^{n}(x, y)
$$

\section{Calculus of the membrane frequencies}


The next step is to determine the natural frequencies of vibration by solving Eq. 1 . Considering the expressions in the Eq. 4 and Eq.21 for the membrane deformation and pressure over the membrane, this equation is expressed for each mode as:

$$
\sum_{m, n} T \nabla^{2} \delta_{m}^{n}(x, y) d_{m}^{n}(t)+\sum_{m, n} \rho_{m} t_{h} \omega_{m}^{n 2} \delta_{m}^{n}(x, y) d_{m}^{n}(t)=\sum_{m, n} P_{m}^{n}(x, y) d_{m}^{n}(t)
$$

By multiplying Eq.23 by the vacuum mode $\delta_{u}^{v}$ and the integration over the surface of the rectangular membrane, the following equation is obtained:

$$
\sum_{m, n} \iint_{S_{m}} T \nabla^{2} \delta_{m}^{n} \delta_{u}^{v} d_{m}^{n}(t) d S+\sum_{m, n} \rho_{m} t_{h} \omega_{m}^{n 2} \iint_{S_{m}} \delta_{m}^{n} \delta_{u}^{v} d_{m}^{n}(t) d S=\sum_{m, n} \iint_{S_{m}} P_{m}^{n} \delta_{u}^{v} d_{m}^{n}(t) d S
$$

The next step is to apply a collocation method, for which the membrane is divided into $N \times N$ rectangular panels. In the center of each panel or the collocation points, the equation (2.23) is fulfilled, and considering the Eq. 22 for the pressure mode on the membrane and the orthogonality condition for the integral expressions, that are zero except for $m=u$ and $n=v$, the following scalar equation is obtained:

$$
\begin{aligned}
& T\left\lfloor\nabla^{2} \delta_{m}^{n}\right\rfloor[\Delta S]\left\{\delta_{u}^{v}\right\} \bar{d}_{m}^{n}+\rho_{m} t_{h} \omega_{m}^{n 2}\left\lfloor\delta_{m}^{n}\right\rfloor[\Delta S]\left\{\delta_{u}^{v}\right\} \bar{d}_{m}^{n}= \\
& =\rho_{f} \frac{\cot h\left(\lambda_{m}^{n} H\right)}{\lambda_{m}^{n}} \omega_{m}^{n 2}\left\lfloor\delta_{m}^{n}\right\rfloor[\Delta S]\left\{\delta_{u}^{v}\right\} \bar{d}_{m}^{n}
\end{aligned}
$$

Where $[\Delta S]$ is a matrix containing the surface area of each rectangular panel.

Making a variation of $m, n, u, v=1,2, \ldots \ldots \ldots . ., N_{m n}$ the following system is obtained leading to an eigenvalue problem:

$$
\left[[K]-\omega^{2}\left([M]+\left[M_{F}\right]\right)\right]\{\bar{d}\}=\{0\} .
$$

where the elements of the three matrices are calculated:

$$
\begin{aligned}
& K_{m u}^{n v}=T\left\lfloor\nabla^{2} \delta_{m}^{n}\right\rfloor[\Delta S]\left\{\delta_{u}^{v}\right\} . \\
& M_{m u}^{n v}=-\rho_{m} t_{h}\left\lfloor\delta_{m}^{n}\right\rfloor[\Delta S]\left\{\delta_{u}^{v}\right\} . \\
& M_{F m u}^{n v}=\rho_{f} \frac{\cot g h\left(\lambda_{m}^{n} H\right)}{\lambda_{m}^{n}}\left\lfloor\delta_{m}^{n}\right\rfloor[\Delta S]\left\{\delta_{u}^{v}\right\} .
\end{aligned}
$$

Once the natural frequencies of the coupled fluid-structure are determined, the wet modes can be computed by determining the eigenvector $\left\{\bar{d}_{i}\right\}$ and expressed as a linear combination of the normal modes of the membrane in vacuum.

It should be noted that only the asymmetric modes are considered because of the fluid incompressibility condition.

\section{Results}


For the verification of the method, in Table 1, the frequencies of the bottom rectangular plate of a rectangular container filled with fluid, $f_{l m}^{n}$ are compared with the results of Kwak [16], that analyzes the Navmi factor of a thin rectangular plate in an aperture of an infinite rigid plane wall in contact with water (incompressible fluid) on one side, calculating the change in the natural frequencies with respect to the vacuum case $f_{v m}^{n}$. In both cases the rectangular plate is considered as simply supported. Consequently, the whole procedure is similar to the one of the membrane and Eq. 1 is replaced by the equation of motion of the plate, from Wang [14]:

$$
D \nabla^{4} \delta(x, y, t)+\rho_{m} t_{h} \frac{\partial^{2} \delta}{\partial t^{2}}=p(x, y, t) .
$$

With $D=\frac{E \cdot t_{h}^{3}}{12\left(1-v^{2}\right)}$ the flexural rigidity, $E$ the elasticity modulus of the material, $t_{h}$ the plate thickness, $v$ the Poisson modulus and $\rho_{m}$ the material density of the plate.

The deformation of the plate is expressed as a linear combination of the corresponding vacuum modes $\Delta_{m}^{n}$ that have the general expression, see Wang [14]: $\delta_{m}^{n}(x, y)=\sin \left(\frac{m \pi x}{a}\right) \cdot \sin \left(\frac{n \pi y}{b}\right)$.

The frequencies $f_{l m}^{n}$ and $f_{v m}^{n}$ are associated to the mode $\delta_{m}^{n}$ of the plate in contact with water and in vacuum, both expressed in $\mathrm{Hz}$.

As it can be observed in Table 1, there is a slight difference in the results of the present method compared to those obtained by Kwak [16], depending on the mode considered for the different values of modal parameters $m$ and $n$, so that the validity of this method can be concluded.

It must be said that the case studied by Kwak is not exactly the same as the one considered here because the rectangular plate is situated in an aperture of an infinite rigid plane wall in contact with water on one side and not in the bottom of a rectangular container filled with water as in the present case. Furthermore, it can be observed a great reduction in frequency in the wet case (with water) with respect to the vacuum case, and this reduction is higher the lower the value of the mode considered, see Gascón-Pérez [17,18].

Table 1. Natural frequencies $(\mathrm{Hz})$ for the bottom simply supported steel plate (in vacuum and water) of a rectangular container filled with water (present method), and in an aperture of an infinite rigid plane wall in contact with water on one side (Kwak case), of side lengths $a=4 \mathrm{~m}, \quad b=5 \mathrm{~m}$, container height $H=3 \mathrm{~m}$ and thickness $t_{h}=1 \mathrm{~cm}$

\begin{tabular}{|ccccc|}
\hline$m, n$ & $f_{v}$ & $f_{l}($ Kwak) & $\begin{array}{c}f_{l} \text { (present } \\
\text { method) }\end{array}$ & $\begin{array}{c}\text { Relative } \\
\text { discrepancy } \\
(\%)\end{array}$ \\
\hline 1,2 & 5.40 & 1.52 & 1.74 & 13.1 \\
2,1 & 7.04 & 2.16 & 2.41 & 10.6 \\
2,2 & 9.96 & 3.39 & 3.68 & 7.78 \\
\hline
\end{tabular}

The next results are obtained for the case of an aluminium bottom membrane of a rectangular container filled with air considered as an incompressible fluid, and the following reference characteristics are considered:

Side lengths $a=0.5 \mathrm{~m}, \quad b=0.4 \mathrm{~m}$, membrane thickness $t_{h}=0.1 \mathrm{~mm}$, container height $H=0.5 \mathrm{~m}$, membrane tension $T=100 \frac{\mathrm{N}}{\mathrm{m}}$, material density $\rho_{m}=2700 \frac{\mathrm{kg}}{\mathrm{m}^{3}}$ 
Fig. 2 shows the frequency variation of the membrane $f_{m}^{n}$ with the relative height of the container $h / H$, for different modes, with $m, n=1,2$.

It can be seen from Fig.2, that the frequency remains constant with the relative height variation and as it approaches to $h$ / $H=0$ decreases quickly at a certain threshold value of the height ratio, depending of the mode considered, indicating that the fluid mass damping effect (the fluid mass matrix) increases a lot by causing the wet natural frequency to decrease ; this value is lower, the higher the value of the mode considered. This qualitative behaviour of frequency with height ratio is similar to that obtained by Tariverdilo et al. $[12,13]$.

Fig. 3 shows the frequency parameter $\Omega$ defined as $\Omega=\sqrt{\frac{\rho_{m} t_{h}}{T}} a \cdot \omega$, as function of the length $a$ of the side of the rectangular membrane, for different modal parameters $m, n$, ie $\Omega_{m}^{n}$, taking into account that the ratio $\frac{a}{b}$ is maintained constant, where $b$ is the other side length. As it can be seen from this Figure, the frequency parameter decreases with the side length and the ratio of decrease is higher the lower the values of the side length. Although for the vacuum case, the frequency parameter remains constant with the side length, and these values are represented in the same Figure.

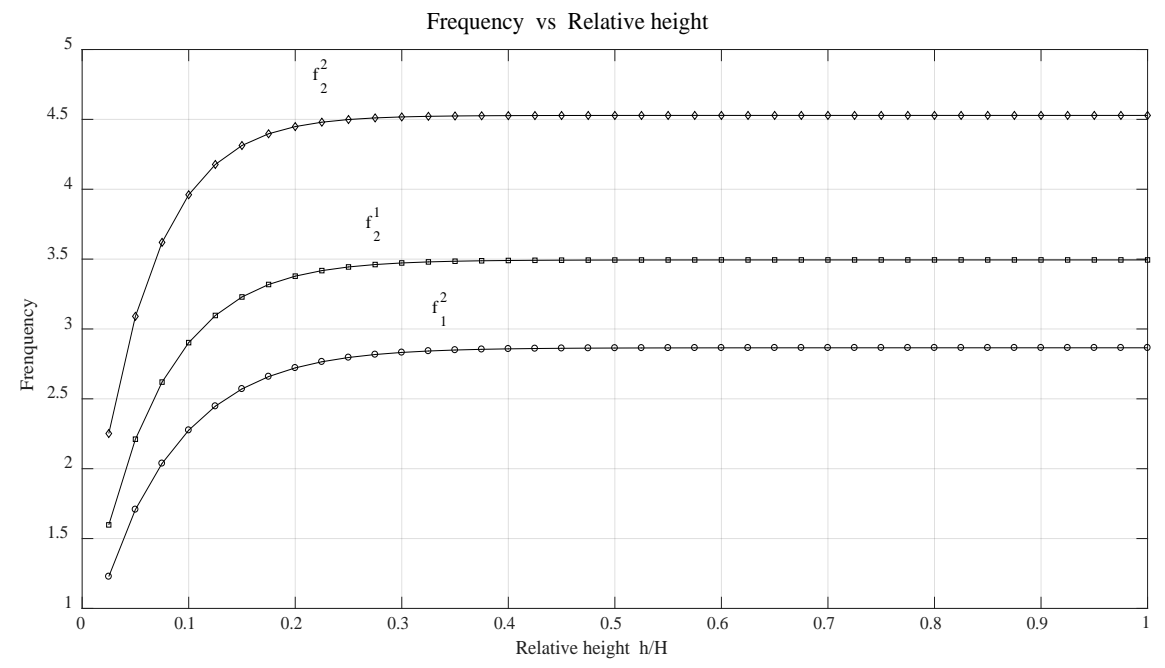

Fig. 2. Variation of membrane frequency $(\mathrm{Hz})$ with the relative height $h / H$ of the rectangular container for different modes $m, n$ 


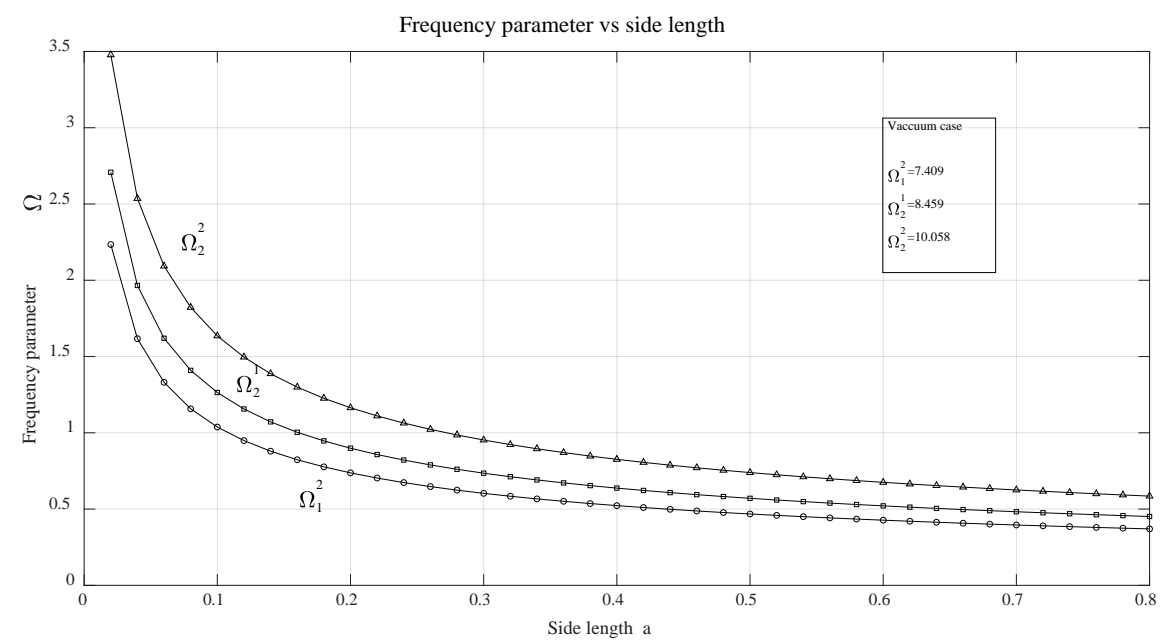

Fig. 3. Variation of membrane frequency parameter $\Omega$ with fluid and in vacuum, with the length of the side of the rectangular membrane for different modes

Fig. 4 shows the frequency parameter $\Omega$ as function of the rectangular membrane aspect ratio $\frac{b}{a}$ in the range $[0.1-1]$, for different values of the modal parameters $m, n$. As it can be seen from the Figure, the frequency parameter decreases with the aspect ratio and the rate of decrease is higher the lower the values of $\frac{b}{a}$.

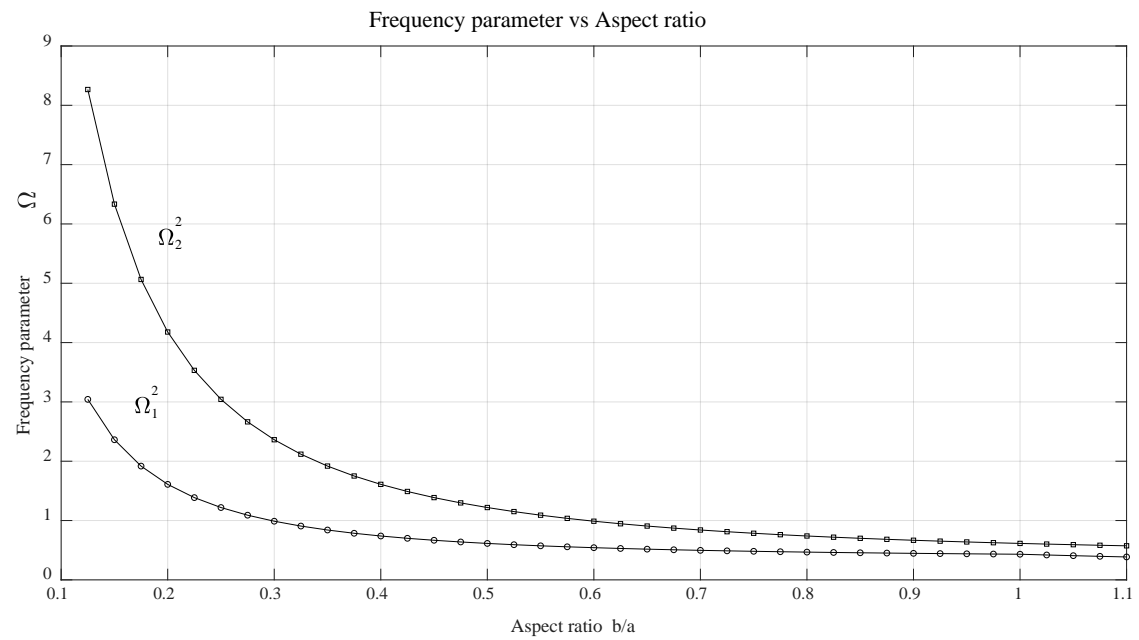

Fig. 4. Variation of membrane frequency parameter $\Omega$ with fluid, with the membrane aspect ratio for different modes

Fig. 5 shows the frequency parameter $\Omega$ as function of the relative thickness $\frac{t}{t_{h}}$ of the membrane, with $\frac{t}{t_{h}}=0$ for zero thickness, for different modes. It can be observed a great reduction in the frequency parameter when the thickness tends to a small value. Also in the same figures, the frequency parameter for the membrane in vacuum is presented. In this case the parameter is independent of the membrane thickness, therefore the figures show the effect of the relative thickness of the membrane coupled with fluid on this coefficient. 


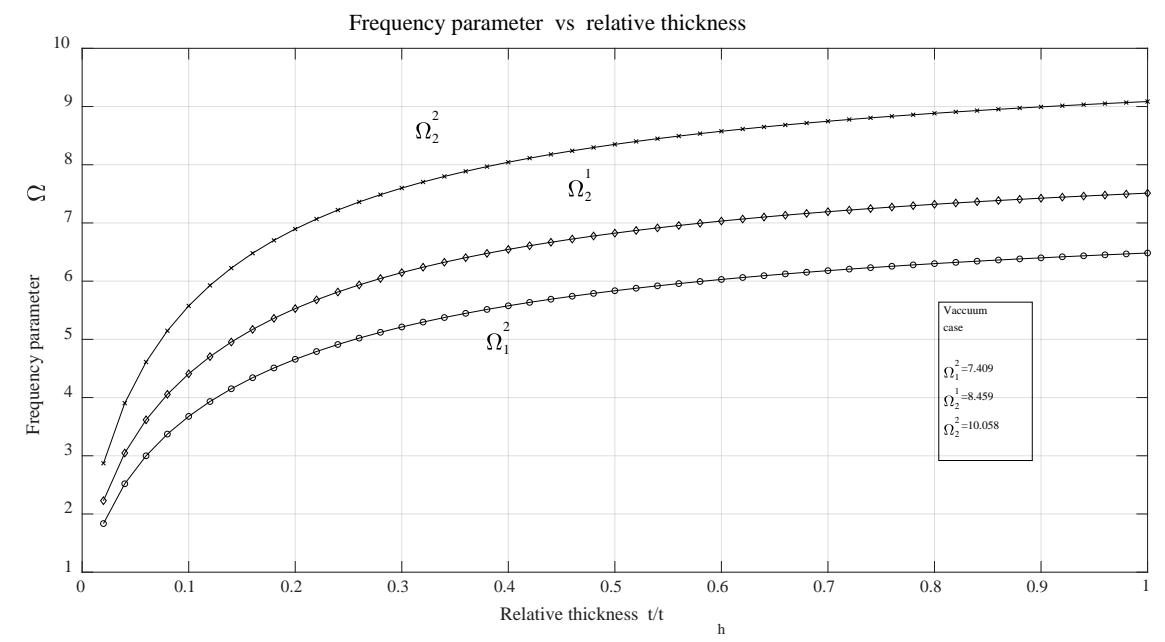

Fig. 5. Variation of membrane frequency parameter $\Omega$ with relative thickness of the membrane for different modal parameters

Fig. 6 shows the frequency parameter $\Omega$ as a function of the fluid density for different modes. It has been taken a density variation from 0 to $300 \mathrm{~kg} / \mathrm{m}^{3}$. This parameter decreases with the fluid density, and the decrease rate is higher the lower the values of it. Furthermore, in the same figure appears the frequency parameter of the membrane in vacuum (absence of fluid), and is the corresponding to the fluid density value of $\rho_{f}=0 \mathrm{~kg} / \mathrm{m}^{3}$.



Fig. 6. Variation of membrane frequency parameter $\Omega$ with fluid density for different modal parameters

It must be said that the frequency parameter remains constant with the variation of the membrane tension, both in the vacuum and in the wet case (with fluid).

Finally, Table 2. shows the frequencies of vibration of the bottom membrane of a rectangular container filled with fluid (air and water) for the different modes of the membrane, and are compared with respect to the values in the vacuum case. The properties of the bottom membrane of the rectangular container, such as height, side dimensions, membrane thickness, tension and material, are the above considered. As it can be observed, there is a great difference, with respect to the vacuum case, in the reduction of the frequency, depending of the fluid considered . This reduction is a little higher, the lower the value of the mode considered. 
Table 2. Natural frequencies (Hz ) for the aluminum membrane of a rectangular container filled with air and water and in the vacuum case for different modes ,with side lengths $a=50 \mathrm{~cm}$ and $b=40 \mathrm{~cm}$, container height $H=50 \mathrm{~cm}$ and membrane thickness $t_{h}=0.1 \mathrm{~mm}$

\begin{tabular}{|cccccc|}
\hline \multirow{2}{*}{$m, n$} & $f_{v}$ & $f_{a}$ & $f_{l}$ & \multicolumn{2}{c|}{ Frequency reduction (\%) } \\
& & & & air & water \\
\hline 1,2 & 44.83 & 39.72 & 2.87 & 11.4 & 93.6 \\
2,1 & 51.26 & 46.02 & 3.49 & 10.2 & 93.2 \\
2,2 & 61.05 & 55.66 & 4.53 & 8.83 & 92.6 \\
2,3 & 75.15 & 69.04 & 6.09 & 8.13 & 91.9 \\
3,2 & 81.79 & 75.62 & 6.92 & 7.54 & 91.5 \\
\hline
\end{tabular}

\section{Conclusions}

The problem of interaction of an structure with fluid has been studied for the case of a bottom membrane of a rectangular container filled with an incompressible and inviscid fluid, as it is in the case of water. In this regard, the fluid velocity potential is solved by a method of separation of variables, and the pressure field is obtained with the momentum's linearized equation, so that considering the membrane equation of motion and its associated work equation, the membrane frequencies of vibration are calculated. For validation of this method, the results are compared with other methods showing a good correlation between them. The influence of different parameters of the rectangular membrane in its dynamic behavior has been studied, in particular the variation of the frequency parameter with the container dimensions, aspect ratio, relative thickness of the membrane and fluid density. Moreover, it has been obtained the variation of the frequency with the height ratio of the rectangular container. Finally, results are presented for the membrane of the rectangular container filled with water and air and compared with respect to the vacuum case.

\section{References}

[1] C.H.M . Jenkins \& U.A. Korde, Membrane vibration experiments: An historical review and recent results, Journal of Sound and Vibration. 295 (2006) 602-613

[2] D.J. Laser \& J.G. Santiago, A review of micropumps, Journal of Micromechanics and Microengineering. 14(6) (2004) 35-64.

[3] L.S. Pan, T.Y. Ng, X.H. Wu, et al, Analysis of Valveless Micropumps with Inertial Effects, Journal of Micromechanics and Microengineering. 13 (2003) 390-399.

[4] R. Shabani, F.G. Golzar, S. Tariverdilo, H. Taraghi, et al, Hydroelastic vibration of a circular diaphragm in the fluid chamber of a reciprocating micro pump, International Journal of Engineering. 27 (2013) 643-650

[5] X. Jin, I. Ladabaum, et al, Fabrication and characterization of surface micromachined capacitive ultrasonic immersion transducers, IEEE ASME Journal of Microelectromechanical Systems. 8(1) (1999) 100-114

[6] E. Defay, C. Millon, C. Malhaire, et al, PZT Thin Films Integration for the Realisation of a High Sensitivity Pressure Microsensor Based on a Vibrating Membrane, Sensors and Actuators. 99 (2002) 64-67.

[7] Y.K. Cheung, \& D. Zhou, Coupled vibratory characteristics of a rectangular container bottom plate, Journal of Fluids and Structures. 14 (2000) 339-357 
[8] F. Daneshmand \& E. Ghavanloo, 2010, Coupled free vibration analysis of a fluid-filled rectangular container with a sagged bottom membrane, Journal of Fluids and Structures. 26 (2010) 236-252

[9] Bauer,H.F., 1981, "Hydroelastic vibrations in a rectangular container ", International Journal of Solids

Structures 17, 639-652

[10] Bauer,H.F., 1995, "Coupled frequencies of a liquid in a circular cylindrical container with elastic liquid

surface cover”, Journal of Sound and Vibration 180, 689-704

[11] Chiba, M., Watanabe, H. \& Bauer H.F., 2002, "Hydroelastic coupled vibrations in a cylindrical container with a membrane bottom containing liquid with surface tension”, Journal of Sound and Vibration

251, 717-740.

[12] Tariverdilo, S., Shahmardani, M., Mirzapour, J. \& Shabani, R., 2013, “Asymmetric free vibration of

circular plate in contact with incompressible fluid”, Applied Mathematical Modelling 37, 228-239.

[13] Tariverdilo, S., Mirzapour, J., Shahmardani, M. \& Rezazadeh, G., 2012, "Free vibration of Membrane/bounded incompressible fluid”, Applied Mathematis and Mechanics 33, 1167-1178.

[14] Wang, C.Y. \& Wang, C.M., 2014, Structural vibration: exact solutions for strings, membranes, beams

and plates, (CRC Press, Boca Raton FL).

[15] Munson, B.R., et al, 2013, “Fundamentals of fluid mechanics” Hoboken, NJ : John Wiley \& Sons, 795p

[16] Kwak, M.K., 1996, "Hydroelastic Vibration of Rectangular Plates”, Journal of Applied mechanics 63,

110-115

[17] M. Gascón-Pérez, Acoustic influence on the vibration of a cylindrical membrane drum filled with a compressible fluid, International Journal of Applied Mechanics. 9(5) 82017)

[18] M. Gascón-Pérez, Interactions of an oscillating rectangular membrane with a compressible fluid, International Journal of Applied Mechanics. 10(2) (2018) 